\title{
THE COUNTABLE CHAIN CONDITION FOR C*-ALGEBRAS
}

\author{
SHUHEI MASUMOTO
}

\begin{abstract}
In this paper, we introduce the countable chain condition for $\mathrm{C}^{*}$ algebras and study its fundamental properties. We show independence from ZFC of the statement that this condition is preserved under the tensor products of $\mathrm{C}^{*}$ algebras.
\end{abstract}

\section{InTRODUCTION}

A topological space is said to have the countable chain condition (CCC for short) if every family of mutually disjoint nonempty open subsets is countable. Any separable space clearly has CCC. Conversely, every metric space which has CCC is separable.

The relation between separability and direct products is simple. The direct product of a family of separable spaces are separable when its cardinality is less than or equal to $2^{\omega}$; but if the cardinality of the family is greater than $2^{\omega}$, then its direct product can be nonseparable. On this point, CCC behaves differently: it is irrelevant to the cardinality of the family. It is known that the direct product of a family of CCC spaces has CCC if for every finite subfamily, its direct product has CCC; however, the statement that the direct product of two CCC spaces has CCC cannot be proved or disproved in ZFC [6, Theorem II.2.24 and Lemma II.4.3].

Now we shall restrict our interest to locally compact Hausdorff spaces. Let $X$ be a locally compact Hausdorff space and $C_{0}(X)$ be the $\mathrm{C}^{*}$-algebra of the continuous functions on $X$ which vanish at infinity. In view of the Gelfand-Naimark theorem, $C_{0}(X)$ contains all the information about the topological structure of $X$. In particular, there is a canonical one to one correspondence between the open sets of $X$ and the closed ideals of $C_{0}(X)$, and CCC can be reformulated as a condition on the ideal structure of $C_{0}(X)$, whence this condition can be generalized for noncommutative $\mathrm{C}^{*}$-algebras. Moreover, since $C_{0}(X \times Y)$ is canonically isomorphic to $C_{0}(X) \otimes C_{0}(Y)$, the discussion on the relation between CCC and direct products yields information about the ideal structure of tensor products of $\mathrm{C}^{*}$-algebras. In this way, we prove the following theorems in this paper:

Theorem 1.1. The minimal tensor product of a family of unital CCC $C^{*}$-algebras has CCC if for every finite subfamily, its minimal tensor product has CCC.

Theorem 1.2. Martin's Axiom, $\mathrm{MA}\left(\omega_{1}\right)$, implies that any minimal tensor product of unital CCC $C^{*}$-algebras has $C C C$.

2010 Mathematics Subject Classification. Primary 47L30; Secondary 03E35, 54A35.

Key words and phrases. Countable chain condition; $\mathrm{C}^{*}$-algebras; tensor products. 
The precise definition of CCC for $\mathrm{C}^{*}$-algebras is introduced in Section 2 In Section 3 Martin's Axiom, which is known to be independent from ZFC, is explained. Here it is also verified that the negation of the Suslin Hypothesis, which is another independent statement explained in Section 3, implies the opposite conclusion of Theorem 1.2. We prove Theorems 1.1 and 1.2 in Section 4 Combining this fact with Theorem 1.1, we conclude that the statement that tensor products of $\mathrm{CCC} \mathrm{C}^{*}$-algebras has CCC is independent from ZFC.

\section{A definition of CCC FOR $\mathrm{C}^{*}$-ALGebras}

Definition 2.1. Two nonzero ideals in a $\mathrm{C}^{*}$-algebra are said to be orthogonal if their intersection is the zero ideal. A $\mathrm{C}^{*}$-algebra has the countable chain condition (CCC) if any family of nonzero mutually orthogonal ideals is countable.

Note that if $\mathcal{I}, \mathcal{J}$ are ideals in a $\mathrm{C}^{*}$-algebra, then $\mathcal{I} \cap \mathcal{J}$ coincides with $\overline{\mathcal{I} \mathcal{J}}$, whence they are orthogonal if and only if $\mathcal{I} \mathcal{J}=0$.

We shall begin with verifying that this definition is a generalization of CCC for topological spaces. Recall that a topological space has CCC if any family of nonempty mutually disjoint open subsets is countable.

Proposition 2.2. Let $X$ be a locally compact Hausdorff space. Then $C_{0}(X)$ has CCC as a $C^{*}$-algebra if and only if X has CCC as a topological space.

Proof. Suppose first that $X$ has CCC and let $\left\{\mathcal{I}_{\lambda}\right\}_{\lambda \in \Lambda}$ be a family of nonzero mutually orthogonal ideals in $C_{0}(X)$. We can take an element $f_{\lambda} \in \mathcal{I}_{\lambda}$ of norm 1 for each $\lambda$. Set $U_{\lambda}=\left\{x \in X|| f_{\lambda}(x) \mid>1 / 2\right\}$. Then $\left\{U_{\lambda}\right\}_{\lambda \in \Lambda}$ is a family of nonempty mutually disjoint open subsets of $X$, whence $\# \Lambda \leq \omega$. Thus, $C_{0}(X)$ has CCC by definition.

If $X$ admits an uncountable family $\left\{U_{\lambda}\right\}_{\lambda \in \Lambda}$ of nonempty mutually disjoint open sets, then $\left\{C_{0}\left(U_{\lambda}\right)\right\}_{\lambda \in \Lambda}$ is an uncountable family of nonzero mutually orthogonal ideals of $C_{0}(X)$. Therefore, $C_{0}(X)$ does not have CCC.

The following easy proposition characterizes CCC. Note that a von Neumann algebra is said to be $\sigma$-finite if it admits no uncountable family of mutually orthogonal projections.

\section{Proposition 2.3.}

(i) Let $\mathcal{A}$ be a $C^{*}$-algebra. Then $\mathcal{A}$ has $C C C$ if and only if there exists no family $\left\{a_{\lambda}\right\}_{\lambda \in \Lambda}$ of nonzero elements such that $a_{\lambda} \mathcal{A} a_{\mu}=0$ for $\lambda \neq \mu$.

(ii) A von Neumann algebra has CCC if and only if its center is $\sigma$-finite.

Proof. (i) Suppose that there is an uncountable family $\left\{a_{\lambda}\right\}_{\lambda \in \Lambda}$ of nonzero elements such that $a_{\lambda} \mathcal{A} a_{\mu}=0$ for $\lambda \neq \mu$. For each $\lambda \in \Lambda$, let $\mathcal{I}_{\lambda}=\overline{\mathcal{A} a_{\lambda} \mathcal{A}}$ be the ideal generated by $a_{\lambda}$. Then $\left\{\mathcal{I}_{\lambda}\right\}_{\lambda \in \Lambda}$ is an uncountable family of nonzero mutually orthogonal ideals, so $\mathcal{A}$ does not have CCC.

Conversely, assume that $\mathcal{A}$ does not have CCC and let $\left\{\mathcal{I}_{\lambda \in \Lambda}\right\}$ be an uncountable family of nonzero mutually orthogonal ideals. Taking nonzero $a_{\lambda} \in \mathcal{I}_{\lambda}$ for each $\lambda$, we obtain $a_{\lambda} \mathcal{A} a_{\mu}=0$ for $\lambda \neq \mu$ because $\mathcal{I}_{\lambda} \mathcal{I}_{\mu}=0$. 
(ii) Let $\mathcal{I}_{1}, \mathcal{I}_{2}$ be ideals of a von Neumann algebra $\mathcal{M}$. Then it can be easily verified that $\mathcal{I}_{1} \mathcal{I}_{2}=0$ if and only if $\overline{\mathcal{I}}_{1}{ }^{\sigma \mathrm{w}} \overline{\mathcal{I}}_{2}{ }^{\sigma \mathrm{w}}=0$, where $\overline{\mathcal{I}}_{i}{ }^{\sigma \mathrm{w}}$ denotes the $\sigma$-weak closure of $\mathcal{I}_{i}$. Now $\overline{\mathcal{I}}_{i}^{\sigma \mathrm{w}}$ is of the form $\mathcal{M} z_{i}$ for a central projection $z_{i}$, and the two ideals are orthogonal if and only if these projections are orthogonal. Therefore, $\mathcal{M}$ has CCC if and only if there is no uncountable family of nonzero mutually orthogonal projections, that is, $\sigma$-finite.

Proposition 2.4. A separable $C^{*}$-algebra has $C C C$.

Proof. Suppose that $\mathcal{A}$ does not have CCC, and $\left\{\mathcal{I}_{\lambda}\right\}_{\lambda \in \Lambda}$ be an uncountable family of nonzero mutually orthogonal ideals. If $h_{\lambda} \in \mathcal{I}_{\lambda}$ is a positive element of norm 1 , then it follows by functional calculus that $\left\|h_{\lambda}-h_{\mu}\right\|=1$. If we denote by $U_{\lambda}$ the open ball of radius $1 / 2$ centered at $h_{\lambda}$, then $\left\{U_{\lambda}\right\}_{\lambda \in \Lambda}$ is an uncountable family of mutually disjoint open subsets. Hence, $\mathcal{A}$ is not separable.

An ideal of a CCC $\mathrm{C}^{*}$-algebra clearly has $\mathrm{CCC}$. Also, it can be easily verified that an extension of a CCC C*-algebra by a CCC $\mathrm{C}^{*}$-algebra has CCC. On the other hand, a quotient of a CCC $\mathrm{C}^{*}$-algebra does not necessarily have $\mathrm{CCC}$. Indeed, let $\beta \mathbb{N}$ be the Stone-Čech compactification of $\mathbb{N}$. It has CCC because it is separable. However, the Stone-Čech remainder $\beta \mathbb{N} \backslash \mathbb{N}$ does not have CCC because there exists an almost disjoint family of $2^{\omega}$ subsets of $\omega$ [6, Theorem II.1.3]. Therefore, $C(\beta \mathbb{N} \backslash \mathbb{N})$ does not have CCC, although it is the quotient of the CCC $\mathrm{C}^{*}$-algebra $C(\beta \mathbb{N}) \simeq \ell^{\infty}$ by $C_{0}(\mathbb{N}) \simeq c_{0}$.

Since $C(\beta \mathbb{N} \backslash \mathbb{N})$ can be obtained as the inductive limit of $\ell^{\infty} \stackrel{\varphi}{\rightarrow} \ell^{\infty} \stackrel{\varphi}{\rightarrow} \cdots$, where $\varphi: \ell^{\infty} \rightarrow \ell^{\infty}$ is defined by $\varphi(f)(n)=f(n+1)$, it also follows that inductive limits of CCC $\mathrm{C}^{*}$-algebras do not necessarily have CCC. On this direction, what we can prove is the following:

Proposition 2.5. Let $\mathcal{A}$ be a $C^{*}$-algebra and $\mathrm{\kappa}$ be an infinite cardinal number with its cofinality not equal to $\omega_{1}$. If there is an increasing sequence $\left\{\mathcal{A}_{\alpha}\right\}_{\alpha<\kappa}$ of CCC $C^{*}$-subalgebras such that $\overline{\bigcup_{\alpha<\kappa} \mathcal{A}_{\alpha}}=\mathcal{A}$, then $\mathcal{A}$ has $C C C$.

To prove this proposition, we use the lemma below. A proof can be found in [3, Lemma III.4.1].

Lemma 2.6. Let $\mathcal{A}$ be a $C^{*}$-algebra and $\left\{\mathcal{A}_{\alpha}\right\}$ be a directed set of subalgebras with its union dense in $\mathcal{A}$. If $\mathcal{I}$ is an ideal of $\mathcal{A}$, then it is obtained as the closure of the union of $\left\{\mathcal{I} \cap \mathcal{A}_{\alpha}\right\}$.

Proof of Proposition 2.5. Assume that there is an uncountable family $\left\{\mathcal{I}_{\lambda}\right\}_{\lambda<\omega_{1}}$ of nonzero mutually orthogonal ideals of $\mathcal{A}$. For each $\lambda$, set

$$
\beta_{\lambda}=\min \left\{\alpha<\kappa \mid \mathcal{I}_{\lambda} \cap \mathcal{A}_{\alpha} \neq 0\right\},
$$

which exists by Lemma 2.6, and write $\beta=\sup _{\lambda} \beta_{\lambda}$. If $\beta<\kappa$ holds, then $\left\{\mathcal{A}_{\beta} \cap \mathcal{I}_{\lambda}\right\}_{\lambda}$ is an uncountable family of nonzero mutually orthogonal ideals, which contradicts to the fact that $\mathcal{A}_{\beta}$ has CCC. On the other hand, if $\beta=\kappa$, then the cofinality of $\kappa$ is $\omega$, whence there is an unbounded increasing sequence $\left\{\gamma_{n}\right\}_{n<\omega}$ in $\kappa$. Now the set $S_{n}$ of $\lambda<\omega_{1}$ with $\beta_{\lambda}<\gamma_{n}$ is at most countable for each $n$, whence $\omega_{1}=\#\left(\bigcup_{n} S_{n}\right) \leq \omega$, a contradiction. 
We close this section by looking at the relation between CCC and von Neumann tensor products. The following proposition, together with results in Section 4 reveals that the situation in the von Neumann algebra setting differs from that in case of $\mathrm{C}^{*}$-algebras.

Proposition 2.7. Let $\mathcal{M}$ and $\mathcal{N}$ be CCC von Neumann algebras. Then the tensor product $\mathcal{M} \bar{\otimes} \mathcal{N}$ of $\mathcal{M}$ and $\mathcal{N}$ as a von Neumann algebra also has CCC.

Proof. We shall denote by $\mathcal{Z}(\mathcal{M}), \mathcal{Z}(\mathcal{N})$ and $\mathcal{Z}(\mathcal{M} \bar{\otimes} \mathcal{N})$ the centers of $\mathcal{M}, \mathcal{N}$ and $\mathcal{M} \bar{\otimes} \mathcal{N}$ respectively. Recall that $\mathcal{Z}(\mathcal{M} \bar{\otimes} \mathcal{N})$ coincides with $\mathcal{Z}(\mathcal{M}) \bar{\otimes} \mathcal{Z}(\mathcal{N})[\overline{9}$, Corollary IV.5.11]. Hence it suffices to show that the tensor product of two abelian $\sigma$-finite von Neumann algebras is also $\sigma$-finite. To see this, note that every abelian von Neumann algebra is of the form $L^{\infty}(\mu)$ for some Radon measure $\mu$ [9, Theorem III.1.18], and it is $\sigma$-finite if and only if $\mu$ is $\sigma$-finite. Since $L^{\infty}(\mu) \bar{\otimes} L^{\infty}(v)$, being canonically isomorphic to $L^{\infty}(\mu \otimes v)$, is $\sigma$-finite if $L^{\infty}(\mu)$ and $L^{\infty}(v)$ are both $\sigma$-finite, the conclusion follows.

A compact Hausdorff space is a stonean space if the closure of every open set is open. Suppose that $X$ is a stonean space and $\mu$ is a Borel measure on it. If for any increasing family $\left\{f_{i}\right\} \in C_{\mathbb{R}}(X)$ with $\sup f_{i}=f \in C_{\mathbb{R}}(X)$ the equality $\mu(f)=\sup \mu\left(f_{i}\right)$ holds, then $\mu$ is said to be normal. A stonean space is called a hyperstonean space if for any nonzero positive $f \in C_{\mathbb{R}}(X)$ there exists a normal Borel measure $\mu$ such that $\mu(f)>0$. It is known that if $X$ is hyperstonean, then $C(X)$ is a von Neumann algebra, and every abelian von Neumann algebra is of this form [9, Theorem III.1.18]. Combining this fact with the preceding proposition, we obtain the following result.

Corollary 2.8. The direct product of two hyperstonean CCC spaces has CCC.

Proof. Let $X, Y$ be hyperstonean CCC spaces. It follows from Proposition 2.7 that the von Neumann tensor product $C(X) \bar{\otimes} C(Y)$ has CCC, and $C(X) \otimes C(Y)$, which is isomorphic to $C(X \times Y)$, is a $C^{*}$-subalgebra of $C(X) \bar{\otimes} C(Y)$. By Proposition 2.3 , it can be easily verified that any $\mathrm{C}^{*}$-subalgebra of commutative $\mathrm{CCC} \mathrm{C} \mathrm{C}^{*}$-algebra has CCC, whence $X \times Y$ has CCC.

\section{Martin's AXIOM ANd SUSLIN's HYPOTHESIS}

In this section, we introduce two statements which are known to be independent from ZFC. Complete treatise for these statements can be found in [6] or [4].

The first statement is Martin's axiom. We shall introduce some definitions related to partially ordered sets in order to express this axiom in a simple form.

Definition 3.1. Let $P$ be a nonempty partially ordered set. Two elements $p, q \in P$ are incompatible if there is no $r \in P$ with $r \leq p$ and $r \leq q$. If there is no uncountable family of mutually incompatible elements in $P$, then $P$ is said to have the countable chain condition (CCC).

As is easily verified, a $\mathrm{C}^{*}$-algebra has $\mathrm{CCC}$ if and only if its nonzero ideals form a CCC partially ordered set, where the order is defined by inclusion. Similarly, 
a nonempty topological space has CCC if and only if the set of nonempty open subsets has CCC as a partially ordered set.

Definition 3.2. Let $P$ be a partially ordered set.

(i) A subset $D \subset P$ is dense if for any $p \in P$ there is $q \in D$ with $q \leq p$.

(ii) A nonempty subset $F \subset P$ is called a filter on $P$ if it satisfies the following:

(a) if $p, q$ are in $F$, then there exists $r \in F$ with $r \leq p$ and $r \leq q$;

(b) if $p \in F$ and $q \geq p$, then $q \in F$.

Suppose that $P$ is a nonempty partially ordered set and fix the topology generated by subsets of the form $\{q \in P \mid q \leq p\}$ for $p \in P$. Then $P$ has CCC if and only if $P$ has CCC as a topological space, and $D \subset P$ is dense if and only if it is dense as a topological subspace.

Now we shall see the exact statement of Martin's axiom MA. Let $\kappa$ be a cardinal number.

$\mathrm{MA}(\kappa)$ : If $P$ is a nonempty CCC partially ordered set and $\left\{D_{\alpha}\right\}_{\alpha \in \kappa}$ is a family of dense subsets in $P$, then there exists a filter $F$ on $P$ such that $F \cap D_{\alpha}$ is not empty for all $\alpha$.

MA: $\mathrm{MA}(\kappa)$ holds for any $\kappa$ with $\omega \leq \kappa<2^{\omega}$.

It is known that $\operatorname{MA}(\omega)$ holds (the Rasiowa-Sikorski lemma) and $M A\left(2^{\omega}\right)$ does not hold in ZFC, whence the Continuum Hypothesis $\mathrm{CH}$ trivially implies MA. On the other hand, MA is indeed consistent with ZFC $+\neg \mathrm{CH}$. In particular,ZFC + $\mathrm{MA}\left(\omega_{1}\right)$ is consistent.

The other statement we use in this paper is Suslin's Hypothesis SH. This hypothesis is related to characterization of the real line as an ordered set. Note that a totally ordered set with the following properties is order-isomorphic to the real line:

(i) unbounded; there does not exist minimum nor maximum element.

(ii) dense; there is an element between any two elements.

(iii) complete; every nonempty bounded subset has a supremum and an infimum.

(iv) separable; there is a countable subset which is dense with respect to the usual order topology.

Definition 3.3. Let $S$ be a totally ordered set which is unbounded, dense and complete. Then $S$ is called a Suslin line if it is nonseparable but CCC as a topological space, where its topology is the usual order topology generated by open intervals.

SH: There does not exist a Suslin line.

In other words, $\mathrm{SH}$ claims that separability in the characterization of the real line above can be replaced by CCC. It is known that the diamond principle $\diamond$, which is a consequence of the axiom of constructibility $\mathbf{V}=\mathbf{L}$, implies $\neg \mathrm{SH}$ [5]. On the other hand, $\mathbf{M A}\left(\omega_{1}\right)$ implies $\mathrm{SH}$, whence $\mathrm{SH}$ is independent from ZFC. 
Proposition 3.4. A Suslin line is a locally compact space.

Proof. It suffices to show that every bounded closed interval is compact. This can be verified by seeing that a proof for the Heine-Borel theorem can be applied to Suslin lines.

Given an open covering $\left\{U_{\lambda}\right\}_{\lambda \in \Lambda}$ of a bounded closed interval $[a, b]$, we shall prove that $[a, b]$ can be covered by finitely many $U_{\lambda}$ 's. Note that we may assume each $U_{\lambda}$ is an open interval.

Let $X$ be the set of all $x \in[a, b]$ such that $[a, x]$ can be covered by finitely many $U_{\lambda}$ 's. Then $X$ is not empty because $a$ is in $X$, and so $\sup X$ exists by completeness. It suffices to show that $\sup X$ belongs to $X$ and coincides with $b$. For this, take $\lambda_{0} \in \Lambda$ such that $\sup X$ is in $U_{\lambda_{0}}$. Then $X \cap U_{\lambda_{0}}$ contains some element, say $c$. Now $[a, c]$ can be covered by finitely many $U_{\lambda}$ 's, and $[c, \sup X]$ is included in $U_{\lambda_{0}}$, so $\sup X$ is in $X$. Also, for any $x \in U_{\lambda_{0}}$, the interval $[a, x]$ can be covered by finitely many $U_{\lambda}$ 's, whence $\sup X$ must coincide with $b$.

The following proposition is from [6, Lemma II.4.3]. For the sake of completeness, we include the proof.

Proposition 3.5. If $S$ is a Suslin line, then $S \times S$ does not have CCC.

Proof. By transfinite induction, we shall take $a_{\alpha}, b_{\alpha}, c_{\alpha} \in S$ for $\alpha<\omega_{1}$ so that

(i) $a_{\alpha}<b_{\alpha}<c_{\alpha}$;

(ii) $b_{\beta} \notin\left(a_{\alpha}, c_{\alpha}\right)$ for $\beta<\alpha$.

This can be carried over because for each $\alpha<\omega_{1}$, the set $\left\{b_{\beta} \mid \beta<\alpha\right\}$, being countable, is not dense in $S$. Setting $U_{\alpha}:=\left(a_{\alpha}, b_{\alpha}\right) \times\left(b_{\alpha}, c_{\alpha}\right)$, we obtain an uncountable family $\left\{U_{\alpha}\right\}_{\alpha<\omega}$ of nonempty mutually disjoint open sets in $S \times S$.

Corollary 3.6. $\neg \mathrm{SH}$ implies the existence of a unital commutative $C C C C^{*}$-algebra $\mathcal{A}$ such that $\mathcal{A} \otimes \mathcal{A}$ does not have $C C C$.

Proof. Let $S$ be a Suslin line and consider the one point compactification $S^{+}$of $S$. Since $S^{+}$contains $S$ as a dense subspace, it is a CCC space. On the other hand, $S^{+} \times S^{+}$does not have CCC because it contains $S \times S$. Now $\mathcal{A}=C\left(S^{+}\right)$is a unital commutative $\mathrm{CCC} \mathrm{C}^{*}$-algebra, but $\mathcal{A} \otimes \mathcal{A}$, being isomorphic to $C\left(S^{+} \times S^{+}\right)$, does not have CCC.

\section{TENSOR PRODUCTS}

Here we shall prove Theorems 1.1 and 1.2. For the first theorem, we need the following combinatorial lemma, which is known as the $\Delta$-system lemma. A proof can be found in any standard textbook on set theory in which the method of forcing is dealt with.

Lemma 4.1 ( $\Delta$-system lemma). Every uncountable family of finite sets includes an uncountable subfamily whose pairwise intersection is constant. 
Proof of Theorem 1.1 Let $\left\{\mathcal{A}_{i}\right\}_{i \in I}$ be a family of unital $\mathrm{C}^{*}$-algebras such that for every finite $J \subset I$, the minimal tensor product $\bigotimes_{i \in J} \mathcal{A}_{i}$ has CCC. We shall prove that $\mathcal{A}:=\bigotimes_{i \in I} \mathcal{A}_{i}$ also has CCC.

Suppose that, contrary to our claim, there exists an uncountable family $\left\{\mathcal{I}_{\lambda}\right\}_{\lambda \in \Lambda}$ of nonzero mutually orthogonal ideals in $\mathcal{A}$. By Proposition 2.6, we can find a finite subset $J_{\lambda} \subset I$ for each $\lambda \in \Lambda$ such that $\mathcal{I}_{\lambda} \cap \bigotimes_{i \in J_{\lambda}} \mathcal{A}_{i} \neq 0$. By the $\Delta$-system lemma, we may assume that there exists a set $R$ such that $J_{\lambda} \cap J_{\mu}=R$ for any $\lambda \neq \mu$.

Since the tensor products are minimal, $\mathcal{I}_{\lambda} \cap \bigodot_{i \in J_{\lambda}} \mathcal{A}_{i}$ is not zero for each $\lambda$, where $\bigodot_{i \in J_{\lambda}} \mathcal{A}_{i}$ is the algebraic tensor products of $\mathcal{A}_{i}$ 's. Take nonzero $f_{\lambda} \in \mathcal{I}_{\lambda} \cap$ $\bigodot_{i \in J_{\lambda}} \mathcal{A}_{i}$ for each $\lambda$. If $R$ is empty, then $f_{\lambda} f_{\mu} \neq 0$ for $\lambda \neq \mu$, which contradicts with the assumption that $I_{\lambda}$ and $\mathcal{I}_{\mu}$ are orthogonal to each other. Therefore, $f_{\lambda}$ is of the form $\sum_{k} g_{\lambda}^{k} \otimes h_{\lambda}^{k}$, where $g_{\lambda}^{k}$ is in $\bigotimes_{i \in R} \mathcal{A}_{i}$ and $\left\{h_{\lambda}^{k}\right\}_{k}$ is a linearly independent set in $\bigotimes_{i \in J_{\lambda} \backslash R} \mathcal{A}_{i}$. If $\lambda \neq \mu$, then the equality $\mathcal{I}_{\lambda} \mathcal{I}_{\mu}=0$ implies that $g_{\lambda}^{k} a g_{\mu}^{l}=0$ for all $k, l$ and $a \in \bigotimes_{i \in R} \mathcal{A}_{i}$. Since for each $\lambda$ there exists $k$ with $g_{\lambda}^{k} \neq 0$, it follows that $\bigotimes_{i \in R} \mathcal{A}_{i}$ does not have CCC by Proposition 2.3, which is a contradiction. Therefore, $\bigotimes_{i \in I} \mathcal{A}_{i}$ has CCC.

Corollary 4.2. Every minimal tensor product of unital separable $C^{*}$-algebras has CCC.

Next, we shall prove the second theorem. For this, we use the following lemma.

Lemma 4.3. Suppose that $\mathcal{A}$ is a CCC $C^{*}$-algebra and $\left\{\mathcal{I}_{\alpha}\right\}_{\alpha<\omega_{1}}$ be a family of its ideals. Then $\mathrm{MA}\left(\omega_{1}\right)$ implies that there exists an uncountable subfamily of the ideals which has the finite intersection property.

Proof. Set $\mathcal{J}_{\alpha}:=\overline{\sum_{\gamma<\alpha} \mathcal{I}_{\gamma}}$. Then $\mathcal{J}_{\alpha}$ is a transfinite decreasing sequence of ideals of $\mathcal{A}$. We shall first show that there exists $\alpha_{0}$ such that $\mathcal{J}_{\beta}$ is an essential ideal of $\mathcal{J}_{\alpha_{0}}$ for all $\beta>\alpha_{0}$. Suppose the contrary. Then we can find an transfinite increasing sequence $\left\{\beta_{\delta}\right\}_{\delta<\omega_{1}} \subset \omega_{1}$ such that the inclusion $\mathcal{J}_{\beta_{\delta+1}} \subset \mathcal{J}_{\beta_{\delta}}$ is not essential. In other words, there exists a nonzero ideal $\mathcal{K}_{\beta_{\delta}}$ of $\mathcal{J}_{\beta_{\delta}}$ such that $\mathcal{K}_{\beta_{\delta}} \cap \mathcal{J}_{\beta_{\delta+1}}=0$. Now $\left\{\mathcal{K}_{\beta_{\delta}}\right\}_{\delta<\omega_{1}}$ is an uncountable family of mutually orthogonal ideals in $\mathcal{A}$, which is a contradiction.

Next, let $P$ be the set of nonzero ideals in $\mathcal{J}_{\alpha_{0}}$. Then $P$ has CCC as a partially ordered set, because an ideal of a CCC C $\mathrm{C}^{*}$-algebra has CCC. For each $\beta>\alpha_{0}$, we set

$$
D_{\beta}=\left\{p \in P \mid p \subset \mathcal{I}_{\gamma} \text { for some } \gamma \geq \beta\right\}
$$

and claim that this is dense in $P$. To prove this, take an arbitrary $q \in P$. Then $q^{\prime}:=q \cap \mathcal{J}_{\beta}$ is not zero by the definition of $\alpha_{0}$. Here, $\mathcal{J}_{\beta}$ is approximated by $\sum_{\gamma \in S} \mathcal{I}_{\gamma}$, where $\left.S \subset\right] \beta, \omega_{1}$ [ is finite. By [8, Theorem3.1.7], $\sum_{\gamma \in S} \mathcal{I}_{\gamma}$ is norm closed for each $S$, whence we can use Lemma 2.6 to conclude that $q^{\prime}$ is the inductive limit of $\left\{q \cap \sum_{\gamma \in S} \mathcal{I}_{\gamma}\right\}_{S}$, and so there exists $\gamma>\beta$ with $q \cap \mathcal{I}_{\gamma} \neq 0$. Since $q \cap \mathcal{I}_{\gamma}$ is clearly in $D_{\beta}$, it follows that $D_{\beta}$ is dense, as desired.

Now let $F$ be a filter on $P$ such that $F \cap D_{\beta}$ is not empty for all $\beta$, whose existence is guaranteed by $\operatorname{MA}\left(\omega_{1}\right)$. Then $\left\{\mathcal{I}_{\alpha} \mid \exists p \in F, p \subset \mathcal{I}_{\alpha}\right\}$ has the finite intersection property, and this is uncountable because the condition $F \cap D_{\beta} \neq \varnothing$ for each $\beta$ 
implies that the set of all $\alpha$ such that $\mathcal{I}_{\alpha} \supset p$ for some $p \in F$ is unbounded in $\omega_{1}$. This completes the proof .

Proof of Theorem 1.2 By Theorem 1.1 it suffices to show that if $\mathcal{A}$ and $\mathcal{B}$ have $\mathrm{CCC}$, then $\mathcal{A} \otimes \mathcal{B}$ has CCC. Assume that, on the contrary, there exists a family $\left\{\mathcal{I}_{\alpha}\right\}_{\alpha<\omega_{1}}$ of nonzero mutually orthogonal ideals in $\mathcal{A} \otimes \mathcal{B}$. Then there exist nonzero ideals $\mathcal{J}_{\alpha} \subset \mathcal{A}$ and $\mathcal{K}_{\alpha} \subset \mathcal{B}$ with $\mathcal{J}_{\alpha} \odot \mathcal{K}_{\alpha} \subset \mathcal{I}_{\alpha}$, by [1] Lemma 2.12 (ii)]. Here, by the preceding lemma, we may assume that $\left\{\mathcal{J}_{\alpha}\right\}_{\alpha}$ and $\left\{\mathcal{K}_{\alpha}\right\}_{\alpha}$ satisfy the finite intersection property. Then, $\mathcal{I}_{\alpha} \cap \mathcal{I}_{\beta}$ contains $\left(\mathcal{J}_{\alpha} \cap \mathcal{J}_{\beta}\right) \otimes\left(\mathcal{K}_{\alpha} \cap \mathcal{K}_{\beta}\right) \neq 0$, which is a contradiction. Therefore, $\mathcal{A} \otimes \mathcal{B}$ has $\mathrm{CCC}$, as expected.

\section{CONCLUding REMARKS AND PROBLEMS}

Let $\mathcal{A}$ be a $\mathrm{C}^{*}$-algebra. By $\operatorname{Prim}(\mathcal{A})$, we shall denote the primitive spectrum of $\mathcal{A}$. (For the definition and elementary properties of primitive spectra, see [7, Chapter 4].) It can be easily verified that $\mathcal{A}$ has $\mathrm{CCC}$ if and only if $\operatorname{Prim}(\mathcal{A})$ has $\mathrm{CCC}$ as a topological space, and Lemma4.3]is obtained as a corollary of [6, Lemma II.2.23]. Here, we may replace $\operatorname{Prim}(\mathcal{A})$ by the prime spectrum prime $(\mathcal{A})$, because the topologies of these spaces are isomorphic as partially ordered sets.

In [10], it is proved that $\operatorname{Prim}(\mathcal{A} \otimes \mathcal{B})$ is homeomorphic to $\operatorname{Prim}(\mathcal{A}) \times \operatorname{Prim}(\mathcal{B})$ provided that either $\mathcal{A}$ or $\mathcal{B}$ is type I. Also, in [1, Proposition 2.17], one can find various conditions for prime $(\mathcal{A} \otimes \mathcal{B})$ to be homeomorphic to prime $(\mathcal{A}) \times \operatorname{prime}(\mathcal{B})$. In these cases, Theorem 1.2 follows from the corresponding fact for topological spaces [6, Theorem II.2.24].

One problem is whether Theorem 1.1 and Theorem 1.2 can be generalized to non-minimal tensor products. Since any tensor product has the minimal tensor product as its quotient, it depends on whether the kernel of the quotient map, which is difficult to be investigated, has CCC.

Another problem lies in the definition of CCC. In this paper we have defined $\mathrm{CCC}$ in terms of ideals, whence this condition is trivial for simple $\mathrm{C}^{*}$-algebras. In order to avoid this phenomenon, we can use hereditary $\mathrm{C}^{*}$-algebras in place of ideals: we shall say two hereditary $\mathrm{C}^{*}$-subalgebras $\mathcal{A}$ and $\mathcal{B}$ are orthogonal to each other if $\overline{\mathcal{A B}}=0$; a $\mathrm{C}^{*}$-algebra has strong $\mathrm{CCC}$ if there is no uncountable family of nonzero mutually orthogonal hereditary $\mathrm{C}^{*}$-subalgebras. Then we can prove the following in the same way as in section 2

- Strong CCC implies CCC.

- $\mathrm{C}^{*}$-subalgebras of a strong $\mathrm{CCC} \mathrm{C}^{*}$-algebra have strong CCC.

- An extension of a strong $\mathrm{CCC} \mathrm{C}^{*}$-algebra by a strong $\mathrm{CCC} \mathrm{C}^{*}$-algebra has strong CCC.

- A von Neumann algebra has strong CCC if and only if it is $\sigma$-finite, so tensor products of two strong CCC von Neumann algebras have strong CCC.

It is expected that conclusions similar to the main theorems of this paper are true, but the author could not prove this. 
Acknowledgement. The author gratitudes to Professor Yasuyuki Kawahigashi, who is my adviser, for several helpful comments on this paper. The author also expresses his thanks to Professor Ilijas Farah, Professor Eberhard Kirchberg, Professor George Elliott, Professor Takeshi Katsura, Alessandro Vignati, Yuki Arano and Yosuke Kubota for many stimulating conversations. This work was supported by the Program for Leading Graduate Schools, MEXT, Japan.

\section{REFERENCES}

[1] E. Blanchard and E. Kirchberg, Non-simple purely infinite $C^{*}$-algebras: the Hausdorff case. J. Funct. Anal. 207 (2004), no. 2, 461-513.

[2] N. P. Brown and N. Ozawa, $C^{*}$-algebras and finite-dimensional approximations. Graduate Studies in Mathematics, 88. American Mathematical Society, Providence, RI, 2008.

[3] K. R. Davidson, $C^{*}$-algebras by example. Fields Institute Monographs, 6. American Mathematical Society, Providence, RI, 1996.

[4] T. Jech, Set theory. The third millenium edition, revised and expanded. Springer Monographs in Mathematics. Springer-Verlag, Berlin, 2003.

[5] R. B. Jensen, The fine structure of the constructible hierarchy. With a section by Jack Silver. Ann. Math. Logic 4 (1972), 229-308; erratum, ibid. 4 (1972), 443.

[6] K. Kunen, Set theory. An introduction to independence proofs Studies in Logic and the Foundations of Mathematics, 102. North-Holland Publishing Co., Amsterdam-New York, 1980.

[7] G. K. Pedersen, $C^{*}$-algebras and their automorphism groups. London Mathematical Society Monographs, 14. Academic Press, Inc. [Harcourt Brace Jovanovich, Publishers], London-New York, 1979.

[8] G. J. Murphy, $C^{*}$-algebras and operator theory. Academic Press, Inc., Boston, MA, 1990.

[9] M. Takesaki, Theory of operator algebras. I. Reprint of the first (1979) edition. Encyclopaedia of Mathematical Sciences, 124. Operator Algebras and Non-commutative Geometry, 5. SpringerVerlag, Berlin, 2002.

[10] A. Wulfsohn, The primitive spectrum of a tensor product of $C^{*}$-algebras. Proc. Amer. Math. Soc. 19 (1968), 1094-1096.

(S. Masumoto) Graduate School of Mathematical Sciences, the University of Tokyo E-mail address: masumoto@ms.u-tokyo.ac.jp 